\title{
Opioid substitution therapy protects against hepatitis $C$ virus acquisition in people who inject drugs: the HITS-c study
}

\section{Bethany White
PhD, MPH \\ Associate Lecturer ${ }^{1,2}$ \\ Gregory J Dore \\ MBBS, FRACP, PhD \\ Head, Viral Hepatitis Clinical Research Program \\ Andrew R Lloyd MBBS MD FRACP Director $^{3}$ \\ William D Rawlinson MBBS, PhD, FRACP Senior Medical Virologist 4 \\ Lisa Maher PhD
Maher \\ Head, Viral Hepatitis Epidemiology and \\ Prevention Program? \\ 1 The Kirby Institute, University of \\ New South Wales, \\ Sydney, NSW. \\ 2 Discipline of \\ Addiction Medicine \\ Sydney Medical School, \\ University of Sydney, \\ Sydney, NSW. \\ $\mathbf{3}$ Inflammation and Infection \\ Research Centre \\ University of \\ New South Wales, \\ Sydney, NSW. \\ 4 Virology Division, SEALS Microbiology, \\ Prince of Wales Hospital, \\ Sydney, NSW. \\ Imaher@ \\ kirby.unsw.edu.au}

MJA 2014; 201: 326-329 doi: 10.5694/mjal3.00153
T he hepatitis $\mathrm{C}$ virus (HCV) is highly prevalent globally among people who inject drugs (PWID). ${ }^{1}$ Among the estimated 15.9 million PWID worldwide, 10 million are estimated to be HCV antibody-positive, ${ }^{1}$ compared with about three million who are HIV-positive. ${ }^{2}$ Australia has relatively good coverage of harm-reduction initiatives among PWID, ${ }^{3}$ with low HIV prevalence ${ }^{4}$ and incidence. ${ }^{5}$ Conversely, high prevalence and incidence of HCV among Australian PWID have been documented. Between 1995 and 2010, HCV prevalence among needle and syringe program (NSP) attendees nationwide remained between $50 \%$ and $60 \%,{ }^{6}$ and an incidence rate of $44.1 / 100$ person-years was observed in a community-based cohort of PWID in south-western Sydney in 1999-2002.7 However, a reported decline in $\mathrm{HCV}$ infection at the population level ${ }^{8}$ suggests that the epidemiology of HCV among PWID in Australia may be changing. There have been no recent data from community-based cohorts of PWID to evaluate whether population-level reductions in $\mathrm{HCV}$ incidence may be related to a reduction in the PWID population size and reduced risk among individual PWID. Such data would also provide key indicators for evaluating the impact of Australia's National Hepatitis C Strategy. ${ }^{9}$

We examined the acquisition of HCV among PWID enrolled in a prospective observational study based in Sydney, New South Wales. Specifically, we assessed HCV incidence from 2009 to 2011, and risk and protective factors associated with incident infection, including uptake of NSPs and opioid substitution therapy (OST - methadone or buprenorphine).

\section{Abstract}

Objective: To estimate hepatitis C virus (HCV) incidence and identify associated risk and protective factors among people who inject drugs (PWID) in Sydney, New South Wales.

Design, setting and participants: Community-based prospective observational study of serologically confirmed HCV antibody-negative PWID enrolled in six Sydney neighbourhoods located in three distinct regions between 10 November 2008 and 31 October 2011.

Main outcome measures: Serologically confirmed HCV incidence per personyears (py); and self-reported demographic and behavioural risk factors for $\mathrm{HCV}$ infection.

Results: The overall incidence of HCV infection was 7.9/100 py. Risk factors independently associated with incident HCV infection were younger age (adjusted hazard ratio [AHR] for age < 27 years, 5.66; 95\% Cl, 1.69-18.95; $P=0.005$ ) and daily or more frequent injecting (AHR, 4.06; 95\% Cl, 1.15-14.30; $P=0.03$ ). Opioid substitution therapy (OST) was protective against $\mathrm{HCV}$ seroconversion and was associated with a reduced risk of incident infection among those who mainly injected heroin or other opioids (AHR for those not receiving OST while mainly injecting heroin or other opioids, $5.64 ; 95 \% \mathrm{Cl}$, $1.30-24.42 ; P=0.02$ ).

Conclusion: The observed HCV incidence was substantially lower than the incidence of 30.8/100 py observed a decade earlier in a similar NSW-based cohort, suggesting a decline in HCV incidence among PWID. This is likely due to increased coverage of OST, combined with a probable decrease in the population of PWID.

\section{Methods}

The Hepatitis C Incidence and Transmission Study - community (HITS-c) is an ongoing communitybased prospective observational study of HCV-negative PWID, designed to inform future trials of candidate HCV vaccines. Research methods have been described elsewhere. ${ }^{10}$ Briefly, field sites in six neighbourhoods located in three distinct Sydney regions (southwestern, inner and western Sydney) were selected after 10 months of ethnographic fieldwork, which included mapping, observation and community engagement to determine potential eligibility and interest in the study. ${ }^{11}$ Snowball sampling techniques based on social and drug-use networks, including incentives for peer referral and targeted outreach sampling, were employed to identify potentially eligible PWID. ${ }^{12}$ Eligibility criteria for study screening were: age 16 years or older; self-reported HCV antibody-negative or unknown status; injection of drugs in the past 12 months; and willingness to provide contact details. PWID satisfying all inclusion criteria (anti-HCV antibody, hepatitis B surface antigen and antiHIV antibody tests all negative) were considered eligible for HITS-c enrolment for 6 weeks after the screening visit. Those who failed to return within this period were required to repeat the screening process.

Behavioural data and serum samples were collected by research assistants who had experience working with the target population and were trained in venepuncture and pre- and post-test counselling. Data from participants enrolled between 
10 November 2008 and 31 October 2011 who had completed at least one behavioural and serological followup assessment were included in this analysis.

The study protocol was approved by the University of New South Wales Human Research Ethics Committee.

\section{Measures}

Data were collected at screening and baseline visits on demographic characteristics, including age, sex, ethnic background, education, housing and employment status. Risk variables included: incarceration history; recent NSP access; time since first injection; main drug injected in the past 6 months; receptive syringe sharing (ie, reusing another person's used syringe); receptive ancillary injecting equipment sharing; backloading (ie, receiving drug solution into one's own syringe from a syringe used by another person, by removing the plunger and squirting the drug into the syringe); injecting in a public place; pooling money to buy drugs; being injected by another person; having a sex partner who injects drugs; and severity of dependence score. Given that OST is indicated for dependence on opioids but not other drugs, a composite variable was created to directly assess the proportion of participants potentially eligible for, but not currently receiving, OST. Participants specifying opioids (predominantly heroin but also methadone, buprenorphine, morphine or oxycodone) as the main drug injected in the past 6 months and also indicating receiving OST in the past 6 months were the reference group, and were compared with those who had mainly injected opioids but indicated no OST in the past 6 months, and those who had mainly injected a non-opioid drug and indicated no OST.

\section{HCV antibody and RNA testing}

Venous blood samples were screened for HCV antibodies using a standard enzyme-linked immunosorbent assay (ELISA; Abbott Architect) and confirmed using the Monolisa HCV Ag-Ab ULTRA assay (Bio-Rad). Samples were screened for HCV RNA using a quantitative $\mathrm{HCV}$ detection assay (COBAS Ampliprep/COBAS
TaqMan HCV 2.0; Roche). HCV antibody and RNA testing was conducted every 24 weeks.

\section{HCV incident case definitions}

Incident cases included participants who tested HCV antibody-negative at screening and were subsequently positive for $\mathrm{HCV}$ antibody and/or HCV RNA at baseline or follow-up visits. Date of infection was estimated as the midpoint between the last negative and the first positive $\mathrm{HCV}$ antibody result.

\section{Data analysis}

Data were analysed using Stata 12.1 (StataCorp). Cumulative HCV incidence was estimated using the person-time method, where the total number of incident infections observed was divided by the total number of person-years (py) of observation, calculated from the date of screening to the estimated date of infection for incident cases or the date of last follow-up before 31 October 2011 for those without incident HCV infection. The exact binomial method was used to calculate $95 \%$ confidence intervals.

Parametric proportional hazards models were used to assess baseline factors independently associated with $\mathrm{HCV}$ infection. With the exponential survival distribution best suiting our data, hazard ratios (HRs) and corresponding 95\% confidence intervals were produced. Consistent with the broader aim of the study to identify and characterise potential participants in future $\mathrm{HCV}$ candidate vaccine field trials, baseline rather than on-study variables were included in the models. We also conducted an analysis to adjust for a potential clustering effect by recruitment region, but the results did not provide evidence for this adjustment $(P>0.05)$ and we concluded that the use of a random effect model was appropriate.

\section{Results}

By 31 October 2011, 268 PWID had been screened for the study. Of these, 166 were eligible for enrolment, and 156 (94\%) were subsequently enrolled. Of 141 participants enrolled for at least 24 weeks, 127 (90\%) completed

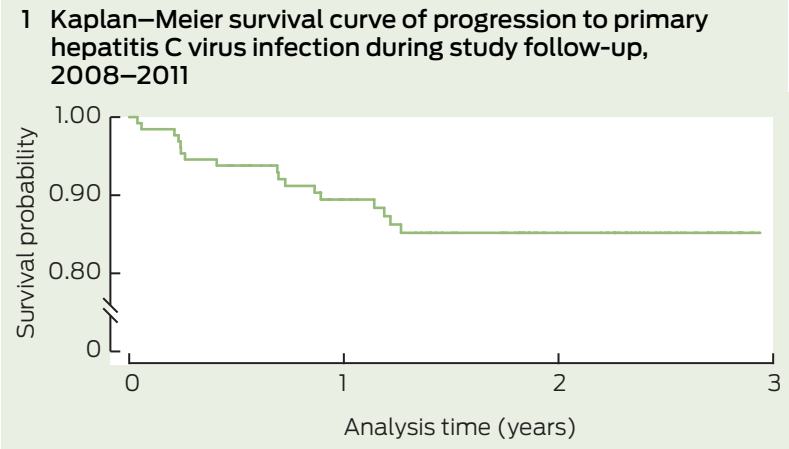

one or more follow-up risk behaviour and serological assessments. An additional two participants who were $\mathrm{HCV}$ antibody-negative at screening and had HCV RNA detected at baseline ( 3 and 6 weeks after screening) were included in the estimates of $\mathrm{HCV}$ incident infection and associated risk factors $(n=129)$.

\section{Incident HCV infection}

In addition to the two participants who were found to be HCV RNA positive at baseline, 15 participants were positive for $\mathrm{HCV}$ antibody and/ or HCV RNA at follow-up visits (six at 24 weeks, five at 48 weeks, and four at 72 weeks). Thus, a total of $17 \mathrm{HCV}$ incident infections were observed over 215.2 py of follow-up, giving an incidence rate of $7.9 / 100$ py $(95 \% \mathrm{CI}$, 4.9-12.7) (Box 1). Annual incidence fluctuated (Box 2). HCV incidence was similar in south-western Sydney $(8.6 / 100$ py) and western Sydney (8.2/100 py), with a slightly lower rate in inner Sydney (5.0/100 py), although this difference was not statistically significant (Appendix; online at mja. com.au).

\section{Risk factors for incident HCV infection}

After controlling a priori for sex and ethnic background, as well as for factors significant at $P<0.05$ in the unadjusted analysis (age, daily or more frequent injecting, receptive syringe sharing and not receiving OST while reporting heroin or other opioids as the main drug injected), incident HCV infection was independently associated with younger age (adjusted HR [AHR] for age less than the sample median of 27 years, 5.66; 95\% CI, 1.69-18.95; $P=0.005)$, daily or more frequent injecting in the 6 months before baseline (AHR, 4.06; 
2 Annual incidence of primary hepatitis C virus infection among 129 study participants, 10 November 2008 - 31 October 2011

\begin{tabular}{lccc} 
Year & $\begin{array}{c}\text { Person-years } \\
\text { at risk }\end{array}$ & $\begin{array}{c}\text { Number newly } \\
\text { diagnosed }\end{array}$ & $\begin{array}{c}\text { Incidence per 100 } \\
\text { person-years (95\% Cl) }\end{array}$ \\
\hline 2009 & 59.9 & 6 & $10.0(4.5-22.3)$ \\
2010 & 88.7 & 6 & $6.8(3.0-15.1)$ \\
$2011^{*}$ & 63.9 & 5 & $7.8(3.3-18.8)$ \\
Total ${ }^{\dagger}$ & 215.2 & 17 & $7.9(4.9-12.7)$ \\
\hline
\end{tabular}

* Includes data only until 31 October 2011. † Includes 2.7 person-years at risk observed between 10 November and 31 December 2008

95\% CI, 1.15-14.30; $P=0.03$ ), and not receiving OST while mainly injecting heroin or other opioids in the past 6 months (AHR, 5.64; 95\% CI, 1.30-24.42; $P=0.02$ ) (Appendix).

\section{Discussion}

The overall HCV incidence we observed of 7.9/100 py between 2009 and 2011 is considerably lower than the incidence of $30.8 / 100$ py in a seronegative cohort of PWID conducted in three sites in NSW a decade earlier, between 1999 and 2001.7 Likewise, our observed incidence of 8.6/100 py in south-western Sydney is lower than the rate of $44.1 / 100$ py found in south-western Sydney in the previous study. ${ }^{7}$ The two studies recruited samples that were similarly marginalised in terms of housing instability, unemployment and history of imprisonment. However, the more recent HITS-c sample was slightly older and had been injecting for longer (consistent with a reduction in new initiates to injecting) and had lower rates of injecting risk behaviour (consistent with reduced incidence of infection). ${ }^{7,13}$ Our HCV incidence rates of $7.9 / 100$ py overall and 5.0/100 py in inner Sydney are also considerably lower than the rate of 18.9/100 py observed in a retrospective cohort of PWID attending an inner Sydney primary health care service two decades ago, in 1992-1993.14 The lower HCV incidence observed in HITS-c than in previous studies is consistent with other data sources indicating that the epidemiology of HCV among PWID in Australia is changing. Declining $\mathrm{HCV}$ incidence over the past decade likely reflects a combination of factors, including changes in the drug market and a decline in the rate of initiation to injecting drug use, a decrease in the PWID population and increased NSP and OST coverage. 8,15

Our study has limitations, including the non-random selection of the sample and the degree to which the results are generalisable to the broader population of PWID. For example, PWID who were likely to refer others to the study may be less likely to engage in receptive syringe sharing, so our results may underestimate true $\mathrm{HCV}$ incidence. However, syringe sharing at baseline was not significantly different between participants who were peer referred and those who were not (data not shown). As behavioural surveys were administered by interviewers, the possibility of misclassification of risk due to the impact of social desirability bias (ie, underreporting of stigmatised or socially undesirable behaviour) cannot be dismissed. Efforts to reduce this included the use of experienced and non-judgemental field staff who were familiar with the study population. Study recall bias was minimised by assessing risk behaviour every 24 weeks. Finally, the small number of incident $\mathrm{HCV}$ infections limited the power of the multivariate analyses and the precision of estimates.

We found that younger age was associated with about a 5.5-fold increased risk of incident $\mathrm{HCV}$ infection. In the previous prospective cohort study of PWID in NSW, injecting for less than 1 year at baseline - a marker of younger age - was associated with an increased risk of incident $\mathrm{HCV}$ infection (AHR, 4.32; 95\% CI, 1.89-9.84). ${ }^{7}$ In the earlier inner Sydney retrospective clinical cohort study, younger age was also associated with $\mathrm{HCV}$ acquisition (adjusted odds ratio for age $<20$ years, $2.47 ; 95 \%$ CI, 1.01-6.01). ${ }^{14}$ In our study, younger participants were less well educated, more likely to indicate residential instability and less likely to be identified as drug-dependent compared with older participants (data not shown). However, age was not associated with differences in key risk behaviour at baseline or follow-up, suggesting that unmeasured factors such as injecting networks and other behavioural, social and structural elements may account for the observed association between age and $\mathrm{HCV}$ acquisition. Daily or more frequent injecting was also independently associated with incident $\mathrm{HCV}$ infection, which may be a marker of increased exposure to injecting with a contaminated syringe.

In our study, OST was protective against incident $\mathrm{HCV}$ infection and associated with a greater than fivefold reduced risk among those who mainly injected heroin or other opioids. There is good existing evidence that stand-alone OST reduces injecting frequency and injecting risk behaviour, as well as growing evidence that OST prevents HIV transmission. ${ }^{16}$ However, previous reports suggest that evidence is limited in relation to $\mathrm{HCV}$ prevention. A recent systematic review of the effectiveness of OST alone in preventing $\mathrm{HCV}$ infection in PWID found pooled results remained non-significant. ${ }^{17}$ The lack of a similarly protective effect for NSPs in our study may be a result of our measure of access, which was restricted to NSP attendance only, whereas individual-level syringe coverage as a proportion of all injections would have been preferable.

Our study is the first communitybased prospective cohort study to observe an independent protective effect of OST against HCV infection among PWID who reported heroin (or other opioids) as the main drug injected. A Welsh study that examined incident $\mathrm{HCV}$ infection (determined using dried blood spot samples) among PWID in 2004-2006 found that participants receiving OST at 12-month follow-up were less likely to acquire $\mathrm{HCV}$ than those not receiving treatment (adjusted incidence rate ratio, $0.34 ; 95 \% \mathrm{CI}, 0.12-0.99$; $P=0.047) .{ }^{18}$ Importantly, when the results of this study were included in an analysis of pooled data from other United Kingdom studies, PWID receiving OST had $64 \%$ reduced odds 
of primary $\mathrm{HCV}$ infection compared with those not receiving OST. ${ }^{19}$

The potential role of OST in preventing $\mathrm{HCV}$ infection is encouraging, given improving access in the Australian setting. The number of people receiving OST nationally has almost doubled since 1998, from 1.3 to 2.1 per 1000 population, ${ }^{20}$ and this has been accompanied by a concurrent reduction in the population size of PWID. ${ }^{8}$ These two factors are the likely key drivers of reduced $\mathrm{HCV}$ incidence, including the lower incidence observed in our study.

A primary goal of Australia's Fourth National Hepatitis C Strategy 2014-2017 is to reduce the transmission of $\mathrm{HCV}$, with the annual incidence of HCV among PWID a key indicator of this goal. ${ }^{9}$ Our results suggest that a reduction in $\mathrm{HCV}$ incidence in the HITS-c cohort may be a result of improved coverage of primary prevention measures such as OST, a priority action area of the Strategy. Our findings are also consistent with international reports of declining $\mathrm{HCV}$ incidence among PWID in high-income settings, including in the Netherlands between 1985 and 2005,21 and the United States between 1998 and 2008. ${ }^{22}$ However, despite a reduction in $\mathrm{HCV}$ incidence, our data suggest that young PWID in Australia remain at risk of $\mathrm{HCV}$ infection. Other prevention and education initiatives, such as increased access to sterile injecting equipment and innovative health service delivery models, will remain essential. Finally, despite recent advances in direct-acting antiviral therapies for $\mathrm{HCV}$ infection and their potential impact on trends in HCV incidence, ${ }^{23}$ barriers to access and prohibitive costs mean that uptake of these new agents by PWID is likely to remain low in the short to medium term. ${ }^{24}$
Acknowledgements: We thank the study participants and their communities for their time and commitment to the study. For recruiting and interviewing participants, we are grateful to Anna Bates, Jarliene Enriquez, Sammy Chow, Ju Park, Len Liao and Aylza Donald. For logistic support for specimen handling, we thank Suzy Teutsch, Hui Li, Brendan Jacka and Alicia Steller. Thanks also to Handan Wand for statistical advice and Carolyn Day for comments on earlier drafts of the manuscript. This study was initially funded by the University of New South Wales (UNSW Hepatitis C Vaccine Initiative) and subsequently by a National Health and Medical Research Council (NHMRC) grant (630483, Hepatitis C Vaccine Preparedness Study). Bethany White was supported by an NHMRC Dora Lush Biomedical Research Postgraduate Scholarship, and Lisa Maher is supported by an NHMRC Senior Research Fellowship. Gregory Dore and Andrew Lloyd are supported by NHMRC Practitioner Fellowships. The Kirby Institute is affiliated with the Faculty of Medicine, UNSW.

Competing interests: No relevant disclosures.

Received 29 Nov 2013, accepted 5 May 2014.

1 Nelson PK, Mathers BM, Cowie B, et al. Global epidemiology of hepatitis B and hepatitis C in people who inject drugs: results of systematic reviews. Lancet 2011; 378: 571-583.

2 Mathers BM, Degenhardt L, Phillips B, et al. Global epidemiology of injecting drug use and HIV among people who inject drugs: a systematic review. Lancet 2008; 372: 1733-1745.

3 Mathers BM, Degenhardt L, Ali H, et al. HIV prevention, treatment, and care services for people who inject drugs: a systematic review of global, regional, and national coverage. Lancet 2010; 375: 1014-1028.

4 Topp L, Day CA, Iversen J, et al; Collaboration of Australian NSPs. Fifteen years of HIV surveillance among people who inject drugs: the Australian Needle and Syringe Program Survey 1995-2009. AIDS 2011; 25: 835-842.

5 Iversen J, Wand H, Topp L, et al. Extremely low and sustained HIV incidence among people who inject drugs in a setting of harm reduction. AIDS 2014: 28: 275-278. doi: 10.1097/ QAD.0000000000000068.

6 Iversen J, Topp L, Maher L Australian NSP Survey national data report 1995-2010. Prevalence of HIV, HCV and injecting and sexual behaviour among NSP attendees. Sydney: The Kirby Institute, University of New South Wales, 2011.

7 Maher L, Jalaludin B, Chant K, et al. Incidence and risk factors for hepatitis C seroconversion in injecting drug users in Australia. Addiction 2006; 101: 1499-1508.

8 Razali K, Thein HH, Bell J, et al. Modelling the hepatitis C virus epidemic in Australia. Drug Alcohol Depend 2007; 91: 228-235.

9 Australian Government Department of Health and Ageing. Fourth National Hepatitis C Strategy 2014-2017. Canberra: Commonwealth of Australia; 2014. http://www.health.gov.au/ internet/main/publishing.nsf/Content/ohpbbvs-hepc (accessed Aug 2014).
10 White B, Dore GJ, Lloyd A, et al. Ongoing susceptibility to hepatitis B virus infection among people who inject drugs in Sydney. Aust N Z J Public Health 2012; 36: 351-357.

11 Maher L, White B, Donald A, et al. Using ethnographic fieldwork to inform hepatitis C vaccine preparedness studies with people who inject drugs. Int J Drug Policy 2010; 21: 194-201.

12 Semaan S, Lauby J, Liebman J. Street and network sampling in evaluation studies of HIV risk-reduction interventions. AIDS Rev 2002; 4 : 213-223.

13 Maher L, Li J, Jalaludin B, et al. High hepatitis C incidence in new injecting drug users: a policy failure? Aust N Z J Public Health 2007; 31: 30-35.

14 van Beek I, Dwyer R, Dore G, et al. Infection with HIV and hepatitis C virus among injecting drug users in a prevention setting: retrospective cohort study. BMJ 1998; 317: 433-437.

15 Iversen J, Wand H, Topp L, et al. Reduction in $\mathrm{HCV}$ incidence among injection drug users attending needle and syringe programs in Australia: a linkage study. Am J Public Health 2013; 103: 1436-1444.

16 MacArthur GJ, Minozzi S, Martin N, et al. Opiate substitution treatment and HIV transmission in people who inject drugs: systematic review and meta-analysis. BMJ 2012; 345: e5945.

17 Hagan H, Pouget ER, Des Jarlais DC. A systematic review and meta-analysis of interventions to prevent hepatitis C virus infection in people who inject drugs. J Infect Dis 2011; 204: 74-83.

18 Craine N, Hickman M, Parry JV, et al. Incidence of hepatitis C in drug injectors: the role of homelessness, opiate substitution treatment, equipment sharing, and community size. Epidemiol Infect 2009; 137: 1255-1265.

19 Turner KM, Hutchinson S, Vickerman P, et al. The impact of needle and syringe provision and opiate substitution therapy on the incidence of hepatitis C virus in injecting drug users: pooling of UK evidence. Addiction 2011; 106: 1978-1988.

20 Australian Institute of Health and Welfare. National opioid pharmacotherapy statistics annual data collection: 2011 report. Canberra: AlHW, 2012. (AlHW Cat. No. HSE 121.)

21 van den Berg C, Smit C, Bakker M, et al. Major decline of hepatitis $C$ virus incidence rate over two decades in a cohort of drug users. Eur $J$ Epidemiol 2007; 22: 183-193.

22 Mehta SH, Astemborski J, Kirk GD, et al. Changes in blood-borne infection risk among injection drug users. J Infect Dis 2011; 203: 587-594.

23 Martin NK, Vickerman P, Grebely J, et al. Hepatitis C virus treatment for prevention among people who inject drugs: modeling treatment scale-up in the age of direct-acting antivirals. Hepatology 2013; 58: 1598-1609.

24 Walsh N, Maher L. HIV and viral hepatitis C coinfection in people who inject drugs: implications of new direct acting antivirals for hepatitis C virus treatment. Curr Opin HIV AIDS 2012; 7: 339-344. 